\title{
Capnography sensor use is associated with reduction of adverse outcomes during gastrointestinal endoscopic procedures with sedation administration
}

\author{
Michael W. Jopling ${ }^{1 *}$ (1) and Jiejing Qiu ${ }^{2}$
}

\begin{abstract}
Background: Evidence to date suggests that capnography monitoring during gastrointestinal endoscopic procedures (GEP) reduces the incidence of hypoxemia, but the association of capnography monitoring with the incidence of other adverse outcomes surrounding these procedures has not been well studied. Our aims were to estimate the incidence of pharmacological rescue events and death at discharge from an inpatient or outpatient hospitalization where GEP was performed with sedation, and to determine if capnography monitoring was associated with reduced incidence of these adverse outcomes.
\end{abstract}

Methods: This retrospective Premier Database analysis included medical inpatients and all outpatients undergoing GEP with sedation. Patients were grouped as follows: (1) pulse oximetry $\left(\mathrm{SpO}_{2}\right)$ only, (2) capnography only, (3) $\mathrm{SpO}_{2}$ with capnography, and (4) neither $\mathrm{SpO}_{2}$ nor capnography. Multivariable logistic regression and propensity-score matching were used to compare patients with capnography sensor use to patients with only $\mathrm{SpO}_{2}$ sensor use. Outcome measures included the incidence of pharmacological rescue events, as defined by administration of naloxone and/or flumazenil, and death.

Results: Two hundred fifty eight thousand and two hundred sixty two inpatients and 3,807,151 outpatients were analyzed. For inpatients, capnography monitoring was associated with a $47 \%$ estimated reduction in the odds of death at discharge (OR: 0.53 [95\% Cl: 0.40-0.70]; $P<0.0001$ ) and a non-significant 10\% estimated reduction in the odds of pharmacological rescue event at discharge $(0.91[0.65-1.3] ; P=0.5661)$. For outpatients, capnography monitoring was associated with a $61 \%$ estimated reduction in the odds of pharmacological rescue event at discharge $(0.39[0.29,0.52] ; P<0.0001)$ and a non-significant $82 \%$ estimated reduction in the odds of death at discharge $(0.18[0.02,1.99] ; P=0.16)$.

Conclusions: In hospital medical inpatients and all outpatients undergoing GEP performed with sedation, capnography monitoring was associated with a reduced likelihood of pharmacological rescue events in outpatients and death in inpatients when assessed at discharge. Despite the limitations of the retrospective data analysis methodology, the use of capnography during these procedures is recommended.

Keywords: Gastrointestinal disorders, Endoscopic procedures, Procedural sedation, Capnography, Patient monitoring, Adverse events

\footnotetext{
* Correspondence: mjopling@jopling.com

${ }^{1}$ NorthStar Anesthesia, Springfield Regional Medical Center, Springfield, OH,

USA

Full list of author information is available at the end of the article
} 


\section{Background}

Gastrointestinal endoscopic procedures (GEP) such as esophagogastroduodenoscopy (EGD), endoscopic retrograde cholangiopancreatography (ERCP), and colonoscopy are standard procedures for the diagnosis and therapy of gastrointestinal disorders, but can be associated with patient discomfort. To improve patient comfort, the use of sedation in these procedures is common. However, the use of sedative agents can result in druginduced airway obstruction, respiratory depression with hypoventilation, and hypoxemia [1-6]. Cardiopulmonary adverse events remain a leading cause of morbidity and mortality during GEP with procedural sedation [3-6].

Given these concerns and potential sequelae, patient monitoring guidelines for procedural sedation have typically recommended continuous pulse oximetry combined with visual assessment of a patient's breathing pattern [7, 8]. Despite the value of these assessments, monitoring of arterial oxygen saturation $\left(\mathrm{SaO}_{2}\right)$ via pulse oximetry does not necessarily provide a satisfactory assessment of the adequacy of ventilation. Importantly, significant alveolar hypoventilation can occur in the presence of normal $\mathrm{SaO}_{2}$ as shown by pulse oximetry $\left(\mathrm{SpO}_{2}\right)$ and inadequate ventilation can precede hypoxemia by several minutes $[1,9,10]$. The risks of procedural sedation are further compounded by the fact that GEP with sedation administration are often conducted without an anesthesia provider present and the delivery of care is performed in a remote hospital location rather than within the primary operating room suite [11].

Given these potential limitations of pulse oximetry and the recognized need for improved patient monitoring during GEP with sedation administration, the use of capnography to monitor end-tidal carbon dioxide $\left(\mathrm{ETCO}_{2}\right)$ is increasingly common $[1,12-14]$. Sidestream and mainstream capnography, by continuously monitoring $\mathrm{ETCO}_{2}$ levels, respiratory rate, and waveform pattern, allow for the near real-time assessment of ventilation in spontaneously breathing patients and provide a more complete assessment of the adequacy of ventilation than either $\mathrm{SpO}_{2}$ or visual inspection of breathing [9, 15-17]. As such, capnography has been clinically demonstrated to provide an earlier indicator of respiratory distress than $\mathrm{SpO}_{2}$ alone $[9,13]$. Several studies have shown that the addition of capnography monitoring during GEP with procedural sedation results in a significant reduction in the incidence of hypoxemia [1, 12-14].

While published evidence to date suggests that capnography monitoring during GEP reduces the incidence of hypoxemia, the association of capnography monitoring with incidence of adverse outcomes when these procedures are performed with sedation has been insufficiently studied. Thus, the aims of this analysis were to estimate the incidence of pharmacological rescue events and death as assessed upon discharge from an inpatient or outpatient hospitalization during which GEP was performed with sedation, separately, for matched patients with and without capnography monitoring using an administrative database.

\section{Methods \\ Patient population}

This was a retrospective analysis of patient data (January 2008 to December 2013) from the Premier Healthcare database (Premier Inc., Charlotte, North Carolina), a privately owned administrative database that is one of the largest hospital-level resource utilization and economic databases in the United States, representing approximately $13 \%$ of inpatient hospitalizations annually [18]. Discharge-level data includes information on patient and provider characteristics, International Classification of Diseases 9th revision Clinical Modification (ICD-9-CM) diagnosis and procedure codes, hospital resource utilization such as specific device usage, medications and laboratory services, discharge disposition (including death), and charges/cost data on all entries. Outcomes for this study were selected based on sedation-related adverse events that could be discretely identified within this retrospective administrative database, and included administration of reversal agents naloxone and/or flumazenil administered on the day of the GEP procedure, and mortality.

Mortality was assessed by identification of patients who were deceased upon hospital discharge. Due to the nature of the retrospective administrative database, causality of mortality cannot be inferred; rather, our intent was to describe the association between GEP under sedation and death at discharge in propensity-matched patient populations.

Data within the Premier database are de-identified in accordance with the Health Insurance Portability and Accountability Act (HIPAA). Per United States Title 45 CFR (Code of Federal Regulations) Part 46.101, institutional review board (IRB) approval for this study was not required under the exemption that this research involved the study of existing data and that the information was recorded in such a manner that the subjects could not be identified, directly or through identifiers linked to the subjects.

Patients were included in the analysis if they were medical inpatients or any outpatients undergoing diagnostic and procedural EGD, ERCP, or colonoscopy as identified using a combination of CPT/ICD-9 codes (see Additional file 1: Table S1). Patients with documentation of sedative medications (propofol, fentanyl, diazepam, meperidine, midazolam, and morphine) were included, while patients who received an inhaled anesthesia agent (isoflurane, desflurane, and sevoflurane) on the 
procedure day were excluded (Fig. 1). Patients who were monitored with capnography and oximetry were identified using medical and surgical supply or respiratory supply billing data indicating that a capnography and/or oximetry sensor was used on and 1-day post-procedure (see Additional file 2: Table S2 for a list of capnography sensor supply codes). For description of the overall population, patients were grouped into four mutually exclusive categories: (1) $\mathrm{SpO}_{2}$ only, (2) capnography only, (3) $\mathrm{SpO}_{2}$ with capnography, and (4) neither $\mathrm{SpO}_{2}$ nor capnography. For the statistical analyses described below, patients were grouped into two mutually exclusive categories: capnography (with and without $\mathrm{SpO}_{2}$ ) and $\mathrm{SpO}_{2}$ only (see Fig. 1).

\section{Statistical analysis}

Descriptive statistics including frequencies and proportions were used for categorical data; means and standard deviations (SD) for continuous variables were calculated for patient demographics and hospital characteristics. Due to the differences between patients with capnography sensor use and patients who had $\mathrm{SpO}_{2}$ sensor use only, the analysis was conducted using Propensity Score (PS) methodology to match patients in order to reduce bias. This matching was done separately for the inpatient and outpatient populations. For the PS matching, a greedy algorithm using the nearest available pair matching method was conducted to match patients on capnography monitoring ( $\pm \mathrm{SpO}_{2}$ monitoring) to those with $\mathrm{SpO}_{2}$ sensor only on a 1 to 1 ratio [19]. The propensity scores were estimated using a logistic regression model adjusted for patient information such as age, gender, race, comorbidities defined by the Charlson Comorbidity Index (CCI) including myocardial infarction, congestive heart failure, dementia, chronic obstructive pulmonary disease (COPD), rheumatoid arthritis, peptic ulcer disease, paralysis, chronic renal failure, cancer, metastatic solid tumor, AIDS, obesity, diabetes, hypertension, peripheral vascular disease, cardiovascular disease, mild liver disease and moderate-severe liver disease, admission type (elective or emergency), and hospital characteristics such as region, bed size, rural vs. urban, and teaching vs. non-teaching (see Additional file 3: Table S3). Due to the large sample size in this analysis $(35,130$

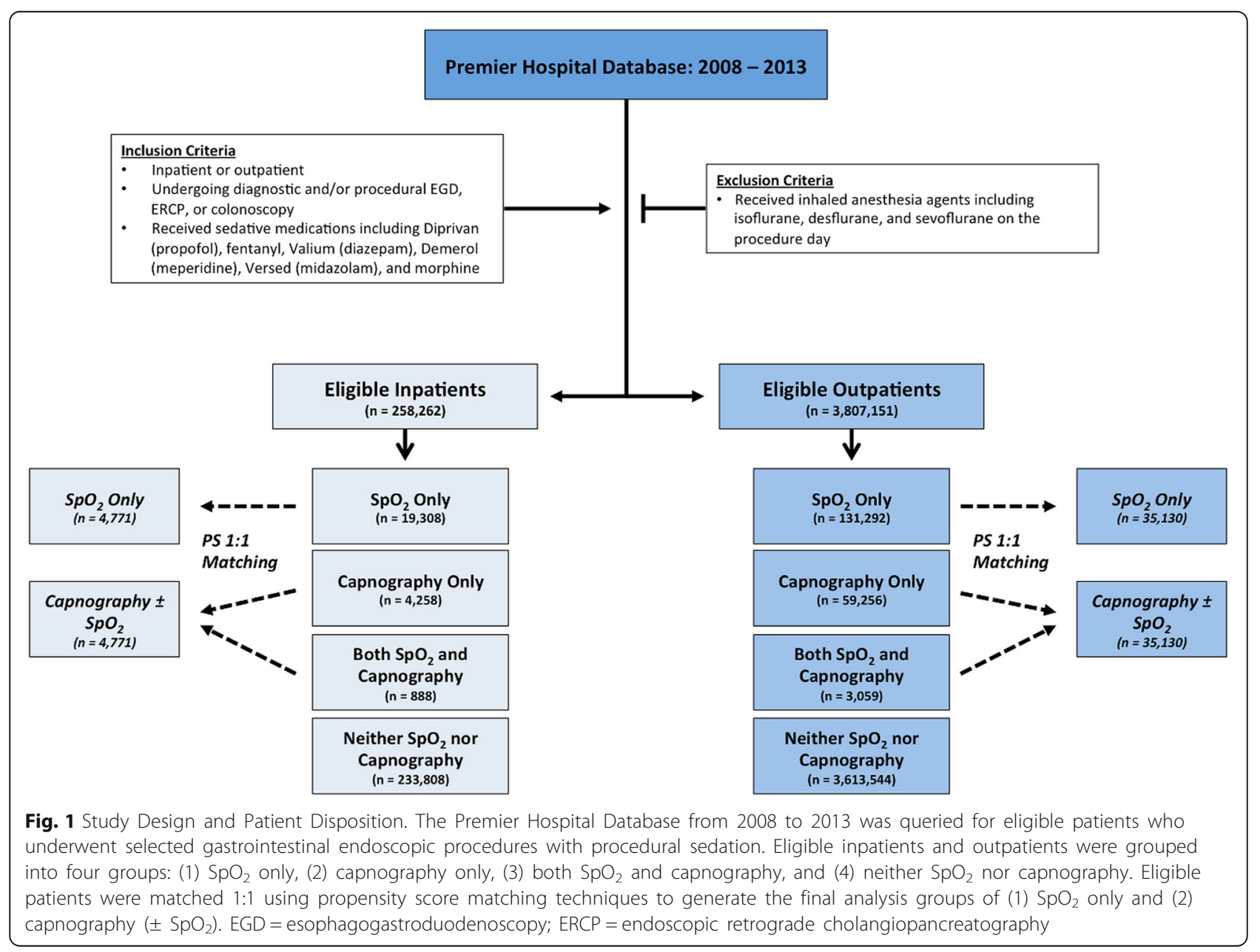


matched outpatient pairs and 4771 matched inpatient pairs), the standardized differences were calculated and compared to a cutoff of 0.1 in order to measure the balance of the matched groups, as this balance measure is not influenced by sample size [20].

After matching, the associations between capnography monitoring and outcomes of interest were estimated using multivariable logistic regression analysis adjusted for all the variables noted above for the PS methodology (see Additional file 3: Table S3), and the association estimates were supported by chi-square tests. These analyses were conducted for the inpatient and outpatient populations separately. The outcomes of interest were pharmacologic rescue events, as defined by administration of naloxone and/or flumazenil, and death, between patients with capnography monitoring $\left( \pm \mathrm{SpO}_{2}\right.$ monitoring) and patients with $\mathrm{SpO}_{2}$ sensor use only, for inpatients and outpatients, separately. The significance level was considered to be 0.05 . Due to the multiple outcomes included in the study, the Bonferroni method was employed to adjust the significance level, therefore a $P$-value less than 0.025 was considered as statistically significant. All statistical analyses were conducted using SAS $^{\circ} 9.2$ for UNIX (Cary, NC, USA).

\section{Results}

\section{Incidence and patient demographics}

Overall, the analysis identified 258,262 eligible inpatients and 3,807,151 eligible outpatients (Fig. 1 and Table 1). For the inpatient population, 19,308 (7.5\%) patients received $\mathrm{SpO}_{2}$ only, 4258 (1.6\%) received capnography only, $888(0.3 \%)$ received both capnography and $\mathrm{SpO}_{2}$, and 233,808 (90.5\%) received neither capnography nor $\mathrm{SpO}_{2}$. For the outpatient population, 131,292 (3.4\%) received $\mathrm{SpO}_{2}$ only, 59,256 (1.6\%) received capnography only, $3059(0.1 \%)$ received both capnography and $\mathrm{SpO}_{2}$, and 3,613,544 (94.9\%) received neither capnography nor $\mathrm{SpO}_{2}$ (Table 1 and Fig. 1).

As expected, the inpatient population tended to be older than the outpatient population (mean age: 64.3 years vs. 57.4 years) with a higher mean CCI (2.53 vs. 0.39). Patients were predominantly white and approximately $50 \%$ male; the most common comorbidities included hypertension, diabetes, and COPD. Most patients were admitted to an urban hospital with $\geq 250$ beds (Table 1 ).

\section{Propensity score matching}

Before matching, the standardized difference scores indicated significant differences $(>0.1)$ in selected patient demographics for both the inpatient and outpatient populations (Additional files 4, and 5: Tables S4 and S5). After matching, there were 4771 matched inpatients and 35,130 matched outpatients who received capnography monitoring ( $\pm \mathrm{SpO}_{2}$ monitoring) or $\mathrm{SpO}_{2}$ monitoring only, and most differences in patient demographics were accounted for by the matching process.

\section{Capnography and the risk of adverse events}

Patient outcomes and multivariable logistic regression analysis for the PS matched patients are presented in Fig. 2 and Additional files 6 and 7: Tables S6 and S7. For the PS matched inpatient population, there were 94 (1.97\%) deaths and 66 (1.38\%) pharmacologic rescue events in patients receiving capnography $\left( \pm \mathrm{SpO}_{2}\right.$ monitoring) as compared to $166(3.48 \%)$ deaths and 74 (1.55\%) pharmacologic rescue events in patients receiving $\mathrm{SpO}_{2}$ monitoring only (Additional file 6: Table S6). For the PS matched outpatient population, there was 1 $(<0.01 \%)$ death and $63(0.18 \%)$ pharmacologic rescue events in patients receiving capnography $\left( \pm \mathrm{SpO}_{2}\right.$ monitoring) as compared to $4(0.01 \%)$ deaths and $148(0.42 \%)$ pharmacologic rescue events in patients receiving $\mathrm{SpO}_{2}$ monitoring only (Additional file 7: Table S7).

Overall, the use of capnography was associated with a $47 \%$ estimated reduction in the odds of death (OR: 0.53 ; 95\% CI: $0.40,0.70 ; P<0.0001)$ for the inpatient population and a $61 \%$ estimated reduction in the odds of pharmacological rescue event (OR: 0.39; 95\% CI: 0.29, 0.52; $P<0.0001$ ) for the outpatient population (Fig. 2). Capnography was also associated with non-significant estimated reductions in the odds of pharmacological rescue event (OR: $0.91 ; 95 \%$ CI: $0.65,1.27 ; P=0.5661)$ for the inpatient population and the odds of death (OR: 0.18 ; 95\% CI: $0.02,1.99 ; P=0.16)$ for the outpatient population (Fig. 2).

Chi-Square analysis of the PS matched samples revealed similar odds ratios as the multivariable logistic regression analysis. More specifically, for the inpatient population, the use of capnography monitoring was associated with a significant estimated reduction in the odds of death (OR: 0.56; 95\% CI: 0.43, 0.72, $P<0.0001$ ) while for the outpatient population, the use of capnography monitoring was associated with a significant estimated reduction in the odds of rescue medication use (OR: 0.42; 95\% CI: 0.32, 0.57; $P<0.0001$ ) (Table 2).

\section{Discussion}

The results of this large database analysis indicate that the use of capnography was associated with a $47 \%$ estimated reduction in the odds of death for the matched inpatient population and a $61 \%$ estimated reduction in the odds of pharmacological rescue event for the matched outpatient population. For the matched inpatient population, the use of capnography was associated with a reduction in both mortality and pharmacologic rescue rates, though reduction in pharmacologic rescue rates was not statistically significant. The reason 
Table 1 Demographics - overall population

\begin{tabular}{|c|c|c|c|c|c|c|c|c|}
\hline \multirow[t]{2}{*}{ Characteristic } & \multicolumn{4}{|c|}{ Inpatient $(n=258,262)$} & \multicolumn{4}{|c|}{ Outpatient $(n=3,807,151)$} \\
\hline & $\begin{array}{l}\mathrm{SpO}_{2} \text { Only } \\
(n=19,308)\end{array}$ & $\begin{array}{l}\text { Capnography Only } \\
(n=4258)\end{array}$ & $\begin{array}{l}\mathrm{SpO}_{2} \text { and } \\
\text { Capnography } \\
(n=888)\end{array}$ & $\begin{array}{l}\text { Neither } \\
(n=233,808)\end{array}$ & $\begin{array}{l}\mathrm{SpO}_{2} \text { Only } \\
(n=131,292)\end{array}$ & $\begin{array}{l}\text { Capnography Only } \\
(n=59,256)\end{array}$ & $\begin{array}{l}\mathrm{SpO}_{2} \text { and Capnography } \\
(n=3059)\end{array}$ & $\begin{array}{l}\text { Neither } \\
(n=3,613,544)\end{array}$ \\
\hline \multicolumn{9}{|l|}{ Age } \\
\hline Overall & $\begin{array}{l}63.43 \pm \\
18.42\end{array}$ & $64.12 \pm 17.19$ & $65.02 \pm 17.05$ & $64.33 \pm 17.52$ & $53.36 \pm 20.91$ & $58.35 \pm 14.70$ & $57.95 \pm 15.24$ & $57.54 \pm 15.31$ \\
\hline $80+$ & 3961 (21\%) & $854(20 \%)$ & 204 (23\%) & $50,707(22 \%)$ & $8260(6 \%)$ & $3249(5 \%)$ & $183(6 \%)$ & $200,883(6 \%)$ \\
\hline $71-80$ & $3886(20 \%)$ & $911(21 \%)$ & $168(19 \%)$ & $47,930(21 \%)$ & $18,818(14 \%)$ & $9147(15 \%)$ & $458(15 \%)$ & $515,643(14 \%)$ \\
\hline $61-70$ & $3733(19 \%)$ & $817(19 \%)$ & $181(20 \%)$ & $43,336(19 \%)$ & $27,376(21 \%)$ & $14,813(25 \%)$ & $729(24 \%)$ & $857,286(24 \%)$ \\
\hline $51-60$ & $3399(18 \%)$ & $731(17 \%)$ & $158(18 \%)$ & $40,155(17 \%)$ & $30,363(23 \%)$ & $16,354(28 \%)$ & $792(26 \%)$ & $\begin{array}{l}1,038,536 \\
(28 \%)\end{array}$ \\
\hline $41-50$ & $2193(11 \%)$ & $517(12 \%)$ & $105(12 \%)$ & $27,244(12 \%)$ & $17,515(13 \%)$ & $8913(15 \%)$ & $520(17 \%)$ & $55,431(15 \%)$ \\
\hline $31-40$ & $1039(5 \%)$ & $237(6 \%)$ & $48(5 \%)$ & $14,190(6 \%)$ & $8924(7 \%)$ & $3852(7 \%)$ & $223(7 \%)$ & $233,897(6 \%)$ \\
\hline $18-30$ & $752(4 \%)$ & $183(4 \%)$ & $17(2 \%)$ & $9253(4 \%)$ & $6412(5 \%)$ & $2597(4 \%)$ & $119(4 \%)$ & $158,524(4 \%)$ \\
\hline$<18$ & $345(2 \%)$ & $8(0 \%)$ & $7(1 \%)$ & $993(0 \%)$ & $13,624(10 \%)$ & $331(1 \%)$ & $35(1 \%)$ & $53,344(1 \%)$ \\
\hline \multicolumn{9}{|l|}{$\mathrm{CCl}$} \\
\hline Overall & $2.59 \pm 2.49$ & $2.53 \pm 2.48$ & $2.57 \pm 2.47$ & $2.52 \pm 2.46$ & $0.51 \pm 1.04$ & $0.45 \pm 0.97$ & $0.79 \pm 1.20$ & $0.39 \pm 0.90$ \\
\hline$>2$ & $8163(42 \%)$ & $1743(41 \%)$ & $368(41 \%)$ & $95,025(41 \%)$ & $6283(5 \%)$ & $2310(4 \%)$ & $228(7 \%)$ & $119,647(3 \%)$ \\
\hline 2 & $2990(15 \%)$ & $637(15 \%)$ & $148(17 \%)$ & $36,636(16 \%)$ & $8371(6 \%)$ & $3302(6 \%)$ & $331(11 \%)$ & $171,185(5 \%)$ \\
\hline 1 & 3722 (19\%) & 894 (21\%) & 169 (19\%) & $49,112(21 \%)$ & $24,123(18 \%)$ & $10,705(18 \%)$ & $824(27 \%)$ & $571,858(16 \%)$ \\
\hline 0 & $4433(23 \%)$ & $984(23 \%)$ & $203(23 \%)$ & $53,035(23 \%)$ & $92,515(70 \%)$ & $42,939(72 \%)$ & $1676(55 \%)$ & $\begin{array}{l}2,750,854 \\
(76 \%)\end{array}$ \\
\hline \multicolumn{9}{|c|}{ APR severity of illness } \\
\hline $1=$ mild & $1751(9 \%)$ & $443(10 \%)$ & $87(10 \%)$ & $23,466(10 \%)$ & $0(0 \%)$ & $0(0 \%)$ & $0(0 \%)$ & $0(0 \%)$ \\
\hline $2=$ moderate & $5621(29 \%)$ & $1428(34 \%)$ & 304 (34\%) & $75,251(32 \%)$ & $0(0 \%)$ & $0(0 \%)$ & $0(0 \%)$ & $0(0 \%)$ \\
\hline $3=$ severe & $8283(43 \%)$ & $1816(43 \%)$ & $360(41 \%)$ & $98,750(42 \%)$ & $0(0 \%)$ & $0(0 \%)$ & $0(0 \%)$ & $0(0 \%)$ \\
\hline 4 = extreme & $3653(19 \%)$ & $571(13 \%)$ & $137(15 \%)$ & $36,341(16 \%)$ & $0(0 \%)$ & $0(0 \%)$ & $0(0 \%)$ & $0(0 \%)$ \\
\hline \multicolumn{9}{|l|}{ Gender } \\
\hline Female & 9975 (52\%) & $2224(52 \%)$ & $450(51 \%)$ & $\begin{array}{l}124,726 \\
(53 \%)\end{array}$ & $74,730(57 \%)$ & $33,635(57 \%)$ & $1867(61 \%)$ & $\begin{array}{l}2,024,108 \\
(56 \%)\end{array}$ \\
\hline Male & $9333(48 \%)$ & $2034(48 \%)$ & $438(49 \%)$ & $\begin{array}{l}109,082 \\
(47 \%)\end{array}$ & $56,562(43 \%)$ & $25,621(43 \%)$ & 1192 (39\%) & $\begin{array}{l}1,589,436 \\
(44 \%)\end{array}$ \\
\hline \multicolumn{9}{|l|}{ Race } \\
\hline White & $\begin{array}{l}14,032 \\
(73 \%)\end{array}$ & 2837 (67\%) & $580(65 \%)$ & $\begin{array}{l}158,011 \\
(68 \%)\end{array}$ & $\begin{array}{l}101,037 \\
(77 \%)\end{array}$ & 45,307 (76\%) & 2689 (88\%) & $\begin{array}{l}2,637,723 \\
(73 \%)\end{array}$ \\
\hline Black & 2756 (14\%) & 731 (17\%) & 177 (20\%) & $34,632(15 \%)$ & $11,813(9 \%)$ & 6380 (11\%) & $284(9 \%)$ & $304,535(8 \%)$ \\
\hline Hispanic & 385 (2\%) & $77(2 \%)$ & 87 (10\%) & 7084 (3\%) & 3987 (3\%) & $1234(2 \%)$ & $0(0 \%)$ & $66,752(2 \%)$ \\
\hline Other & 2135 (11\%) & $613(14 \%)$ & $44(5 \%)$ & $34,081(15 \%)$ & 14,455 (11\%) & $6335(11 \%)$ & $86(3 \%)$ & $604,534(17 \%)$ \\
\hline \multicolumn{9}{|l|}{ Comorbidity } \\
\hline HTN & $\begin{array}{l}12,079 \\
(63 \%)\end{array}$ & 2747 (65\%) & $582(66 \%)$ & $\begin{array}{l}144,259 \\
(62 \%)\end{array}$ & $40,244(31 \%)$ & $18,788(32 \%)$ & 1451 (47\%) & $972,692(27 \%)$ \\
\hline Diabetes & 5930 (31\%) & 1354 (32\%) & 274 (31\%) & 71,495 (31\%) & $17,501(13 \%)$ & 7797 (13\%) & 615 (20\%) & $393,796(11 \%)$ \\
\hline COPD & 4727 (25\%) & 1031 (24\%) & 209 (24\%) & $55,507(24 \%)$ & $11,656(9 \%)$ & $4524(8 \%)$ & 570 (19\%) & $254,671(7 \%)$ \\
\hline CRF & 4070 (21\%) & 873 (21\%) & $183(21 \%)$ & $46,587(20 \%)$ & $1658(1 \%)$ & $593(1 \%)$ & $63(2 \%)$ & $35,807(1 \%)$ \\
\hline $\mathrm{CHF}$ & 3688 (19\%) & 681 (16\%) & $148(17 \%)$ & $41,238(18 \%)$ & $2281(2 \%)$ & 665 (1\%) & $113(4 \%)$ & $33,729(1 \%)$ \\
\hline PUD & 2748 (14\%) & 577 (14\%) & $98(11 \%)$ & $33,639(14 \%)$ & $5453(4 \%)$ & 2045 (4\%) & 162 (5\%) & 111,461 (3\%) \\
\hline Obesity & 2125 (11\%) & 461 (11\%) & 100 (11\%) & 25,189 (11\%) & 6027 (5\%) & 1644 (3\%) & $143(5 \%)$ & $130,982(4 \%)$ \\
\hline Cancer & 1895 (10\%) & 449 (11\%) & 86 (10\%) & $23,155(10 \%)$ & 3970 (3\%) & 1611 (3\%) & $85(3 \%)$ & $85,219(2 \%)$ \\
\hline MLD & 2011 (10\%) & 417 (10\%) & 98 (11\%) & $23,108(10 \%)$ & 1913 (2\%) & 808 (1\%) & $63(2 \%)$ & $39,733(1 \%)$ \\
\hline
\end{tabular}


Table 1 Demographics - overall population (Continued)

\begin{tabular}{|c|c|c|c|c|c|c|c|c|}
\hline \multirow[t]{2}{*}{ Characteristic } & \multicolumn{4}{|c|}{ Inpatient $(n=258,262)$} & \multicolumn{4}{|c|}{ Outpatient $(n=3,807,151)$} \\
\hline & $\begin{array}{l}\mathrm{SpO}_{2} \text { Only } \\
(n=19,308)\end{array}$ & $\begin{array}{l}\text { Capnography Only } \\
(n=4258)\end{array}$ & $\begin{array}{l}\mathrm{SpO}_{2} \text { and } \\
\text { Capnography } \\
(n=888)\end{array}$ & $\begin{array}{l}\text { Neither } \\
(n=233,808)\end{array}$ & $\begin{array}{l}\mathrm{SpO}_{2} \text { Only } \\
(n=131,292)\end{array}$ & $\begin{array}{l}\text { Capnography Only } \\
(n=59,256)\end{array}$ & $\begin{array}{l}\mathrm{SpO}_{2} \text { and Capnography } \\
(n=3059)\end{array}$ & $\begin{array}{l}\text { Neither } \\
(n=3,613,544)\end{array}$ \\
\hline MSLD & 2009 (10\%) & 399 (9\%) & $91(10 \%)$ & $22,687(10 \%)$ & 2193 (2\%) & $917(2 \%)$ & $66(2 \%)$ & $43,521(1 \%)$ \\
\hline $\mathrm{Ml}$ & $1713(9 \%)$ & $348(8 \%)$ & $72(8 \%)$ & $21,179(9 \%)$ & $2171(2 \%)$ & $982(2 \%)$ & $115(4 \%)$ & 49,888 (1\%) \\
\hline CVD & $1303(7 \%)$ & $256(6 \%)$ & $54(6 \%)$ & $15,601(7 \%)$ & $764(1 \%)$ & $183(<1 \%)$ & $36(1 \%)$ & $11,995(<1 \%)$ \\
\hline PVD & $1406(7 \%)$ & $281(7 \%)$ & $63(7 \%)$ & $15,224(7 \%)$ & $1081(1 \%)$ & $340(1 \%)$ & $57(2 \%)$ & $17,958(<1 \%)$ \\
\hline MST & 916 (5\%) & $218(5 \%)$ & $50(6 \%)$ & $11,248(5 \%)$ & $496(<1 \%)$ & $190(<1 \%)$ & $13(<1 \%)$ & $9539(<1 \%)$ \\
\hline RA & $657(3 \%)$ & $144(3 \%)$ & $16(2 \%)$ & $8177(4 \%)$ & $1453(1 \%)$ & $693(1 \%)$ & $60(2 \%)$ & $29,597(1 \%)$ \\
\hline Dementia & $172(1 \%)$ & $24(1 \%)$ & $12(1 \%)$ & $2127(1 \%)$ & $39(<1 \%)$ & $19(<1 \%)$ & $2(<1 \%)$ & $733(<1 \%)$ \\
\hline Paralysis & $172(1 \%)$ & $29(1 \%)$ & $7(1 \%)$ & $2334(1 \%)$ & $91(<1 \%)$ & $23(<1 \%)$ & $6(<1 \%)$ & $1459(<1 \%)$ \\
\hline AIDS & $65(<1 \%)$ & $15(<1 \%)$ & $10(1 \%)$ & $978(<1 \%)$ & $72(<1 \%)$ & $27(<1 \%)$ & $2(<1 \%)$ & $1687(<1 \%)$ \\
\hline \multicolumn{9}{|l|}{ Region } \\
\hline E. N. Central & 707 (4\%) & 864 (20\%) & $19(2 \%)$ & $36,866(16 \%)$ & $900(1 \%)$ & 7311 (12\%) & $30(1 \%)$ & 747,309 (21\%) \\
\hline E. S. Central & 2175 (11\%) & $7(<1 \%)$ & $41(5 \%)$ & $12,738(5 \%)$ & $22,514(17 \%)$ & $113(<1 \%)$ & $1886(62 \%)$ & $218,449(6 \%)$ \\
\hline M. Atlantic & $1097(6 \%)$ & $12(<1 \%)$ & $0(0 \%)$ & $23,470(10 \%)$ & $148(<1 \%)$ & $8(<1 \%)$ & $0(0 \%)$ & 195,389 (5\%) \\
\hline Mountain & $434(2 \%)$ & $370(9 \%)$ & $0(0 \%)$ & $11,684(5 \%)$ & $1029(1 \%)$ & $2959(5 \%)$ & $2(<1 \%)$ & 205,205 (6\%) \\
\hline $\begin{array}{l}\text { New } \\
\text { England }\end{array}$ & $446(2 \%)$ & $108(3 \%)$ & $0(0 \%)$ & $8113(3 \%)$ & $2281(2 \%)$ & 7431 (13\%) & $1(<1 \%)$ & 193,418 (5\%) \\
\hline Pacific & $1584(8 \%)$ & $380(9 \%)$ & $23(3 \%)$ & 34,221 (15\%) & 11,604 (9\%) & $3170(5 \%)$ & $69(2 \%)$ & $572,954(16 \%)$ \\
\hline S. Atlantic & 8637 (45\%) & $2122(50 \%)$ & 745 (84\%) & 67,627 (29\%) & $53,391(41 \%)$ & 31,137 (53\%) & 776 (25\%) & 884,648 (24\%) \\
\hline W. N. Central & 603 (3\%) & $80(2 \%)$ & $8(1 \%)$ & $16,276(7 \%)$ & $8190(6 \%)$ & $355(1 \%)$ & $7(<1 \%)$ & $370,834(10 \%)$ \\
\hline W. S. Central & 3625 (19\%) & $315(7 \%)$ & $52(6 \%)$ & $22,813(10 \%)$ & $31,235(24 \%)$ & $6772(11 \%)$ & $288(9 \%)$ & 225,338 (6\%) \\
\hline \multicolumn{9}{|l|}{ Teaching Hospital } \\
\hline Yes & $8672(45 \%)$ & 804 (19\%) & 130 (15\%) & 88,195 (38\%) & 41,105 (31\%) & 9659 (16\%) & $491(16 \%)$ & $\begin{array}{l}1,117,242 \\
(31 \%)\end{array}$ \\
\hline \multicolumn{9}{|l|}{ Hospital Bed size } \\
\hline$<250$ & $2523(13 \%)$ & 822 (19\%) & $81(9 \%)$ & $52,543(22 \%)$ & 38,376 (29\%) & $17,738(30 \%)$ & 1968 (64\%) & $\begin{array}{l}1303,833 \\
(36 \%)\end{array}$ \\
\hline $250-500$ & 7752 (40\%) & $2571(60 \%)$ & 257 (29\%) & $\begin{array}{l}112,743 \\
(48 \%)\end{array}$ & $25,602(20 \%)$ & 33,685 (57\%) & $1022(33 \%)$ & $\begin{array}{l}1,672,591 \\
(46 \%)\end{array}$ \\
\hline $500+$ & $9033(47 \%)$ & 865 (20\%) & $550(62 \%)$ & $68,522(29 \%)$ & $67,314(51 \%)$ & 7833 (13\%) & $69(2 \%)$ & $637,120(18 \%)$ \\
\hline \multicolumn{9}{|l|}{ Hospital Location } \\
\hline Rural & 2320 (12\%) & $1124(26 \%)$ & $47(5 \%)$ & 26,096 (11\%) & $27,118(21 \%)$ & 8916 (15\%) & $44(1 \%)$ & 598,635 (17\%) \\
\hline Urban & $\begin{array}{l}16,988 \\
(88 \%)\end{array}$ & 3134 (74\%) & 841 (95\%) & $\begin{array}{l}207,712 \\
(89 \%)\end{array}$ & $\begin{array}{l}104,174 \\
(79 \%)\end{array}$ & $50,340(85 \%)$ & 3015 (99\%) & $\begin{array}{l}3,014,909 \\
(83 \%)\end{array}$ \\
\hline
\end{tabular}

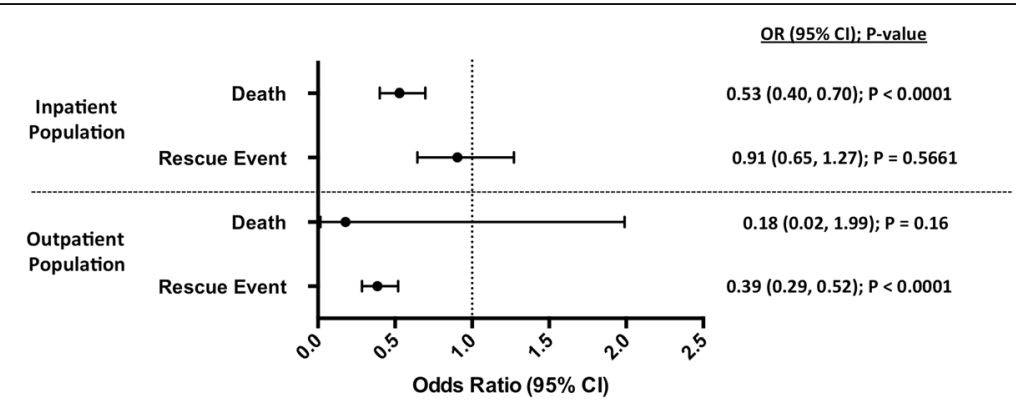

Fig. 2 Propensity Score Matched Odds Ratios. Adjusted Odds Ratios (95\% Cl) for death and rescue event (naloxone and/or flumazenil administration) for capnography $\left( \pm \mathrm{SpO}_{2}\right)$ as compared to $\mathrm{SpO}_{2}$ only, for the matched inpatient and outpatient populations. $\mathrm{Cl}=$ confidence interval; $\mathrm{OR}=$ odds ratio 
Table 2 Chi-Square using PS Matched Samples

\begin{tabular}{|c|c|c|c|c|c|c|}
\hline \multirow{2}{*}{$\begin{array}{l}\text { Patient } \\
\text { population }\end{array}$} & \multicolumn{2}{|c|}{ Outcome } & \multicolumn{2}{|c|}{ Capnography } & \multirow[t]{2}{*}{$P$-value } & \multirow[t]{2}{*}{ OR } \\
\hline & & & Yes & No & & \\
\hline \multirow[t]{4}{*}{ Inpatient } & Death & Yes & $94(2.0 \%)$ & 166 (3.5\%) & $<0.0001$ & $\begin{array}{l}0.56 \\
(0.43,0.72)\end{array}$ \\
\hline & & No & $\begin{array}{l}4,677 \\
(98.0 \%)\end{array}$ & $\begin{array}{l}4,605 \\
(96.5 \%)\end{array}$ & & \\
\hline & $\begin{array}{l}\text { Rescue } \\
\text { Event }\end{array}$ & Yes & $\begin{array}{l}66 \\
(1.4 \%)\end{array}$ & $74(1.6 \%)$ & 0.50 & $\begin{array}{l}0.89 \\
(0.64,1.24)\end{array}$ \\
\hline & & No & $\begin{array}{l}4,705 \\
(98.6 \%)\end{array}$ & $\begin{array}{l}4,697 \\
(98.5 \%)\end{array}$ & & \\
\hline \multirow[t]{4}{*}{ Outpatient } & Death & Yes & ND & ND & ND & ND \\
\hline & & No & & & & \\
\hline & $\begin{array}{l}\text { Rescue } \\
\text { Event }\end{array}$ & Yes & $\begin{array}{l}63 \\
(0.2 \%)\end{array}$ & $\begin{array}{l}148 \\
(0.4 \%)\end{array}$ & $<0.0001$ & $\begin{array}{l}0.42 \\
(0.32,0.57)\end{array}$ \\
\hline & & No & $\begin{array}{l}35,067 \\
(99.8 \%)\end{array}$ & $\begin{array}{l}34,982 \\
(99.6 \%)\end{array}$ & & \\
\hline
\end{tabular}

$N D$ not determined, $O R$ odds ratio

for close rates of pharmacologic rescue in the inpatient setting may be explained by several factors. First, patients with more monitors may be more closely watched by attending clinicians, who may check both alarms and patients. Second, in the inpatient setting, resuscitation may proceed immediately to mask bag ventilation or endotracheal intubation instead of reliance on pharmacologic reversal agents, whereas in the outpatient setting where anesthesia personnel may be limited, pharmacologic reversal may be preferred to primary airway control. Lastly, the fact that the outpatient group demonstrated a reduction in pharmacologic rescue but not death rates may be related to patient flow in the outpatient setting. If a patient's condition declines significantly, they are likely to be admitted to hospital well before death occurs; thus, it is not surprising that mortality is rarely seen in the outpatient setting. However, inpatient admissions from the outpatient setting are not reliably retrievable from this retrospective database, so this analysis is not presented here. Regardless, to our knowledge, these data provide the first evidence that capnography use during GEP with procedural sedation is associated with a reduction in the odds of these adverse outcomes.

Procedural sedation can result in loss of protective pharyngeal airway reflexes, upper airway obstruction, central respiratory depression, alveolar hypoventilation, atelectasis, hypercapnia and hypoxemia [21]. Cardiopulmonary adverse events remain a leading cause of morbidity and mortality with GEP [3-6]. In a study of 21,011 procedures using midazolam and/or diazepam, Arrowsmith et al. reported serious cardiopulmonary complications in 5.4 per 1000 procedures [4]. Similarly, in a database analysis of over 300,000 endoscopic procedures with sedation, Sharma et al. reported an incidence of 9.3 cardiopulmonary adverse events per 1000 procedures [6]. These authors also noted that the presence of the endoscope across the upper airway along with a depressed respiratory drive due to the sedative medications in combination with the inability to accurately assess ventilation using pulse oximetry can result in undetected hypoventilation and a higher incidence of cardiopulmonary adverse events [6]. Importantly, the risks of GEP adverse events exceed those typically reported for general anesthesia procedures performed outside the operating room [11].

Given these risks of procedural sedation and the fact that sedation levels during GEP often approach those of general anesthesia, [2] adequate patient monitoring is critical, especially with these types of procedures often conducted by non-anesthesiologists outside of the operating room, where personnel and equipment availability can limit effective response to acute deterioration. In fact, in an analysis of 63,000 patients undergoing diagnostic or therapeutic procedures under sedation or anesthesia, over $40 \%$ of patients were sedated by non-anesthesia providers and $12.4 \%$ of the anesthesiology cases were performed outside of the operating room [22].

Historically, patient monitoring during GEP has focused on pulse oximetry in combination with vital sign monitoring and visual inspection of ventilation $[7,8]$. Unfortunately, as noted above, monitoring arterial oxygen saturation via pulse oximetry, especially in patients receiving supplemental oxygen therapy, is inadequate for effectively detecting the onset of hypoventilation $[1,9$, $10,23]$. Importantly, several closed claims analyses have indicated that the application of better monitoring, including capnography, could have prevented nearly half of claims associated with oversedation [11, 24]. To date, a number of studies have indicated that the addition of capnography to standard monitoring provides superior detection of respiratory depression during procedural sedation [10, 12, 13, 25-27]. These data and others have led to the recent recognition of capnography as a critical component of adequate patient monitoring during moderate or deep sedation by the American Society of Anesthesiologists (ASA) [28]. In addition, several recent cost-benefit analyses support the use of capnography $[29,30]$. Despite the apparent clinical and economic benefits of capnography and support by the ASA and other governing bodies, the use of capnography in these types of procedures remains relatively low.

\section{Limitations}

We note that this retrospective database analysis has several limitations. While we are able to demonstrate an association between capnography use and a reduction in adverse outcomes, we cannot demonstrate causation. Additionally, we are unable to characterize the training, 
education, experience and expertise of those monitoring, interpreting and acting upon the results of monitoring. Finally, with an administrative database, we are unable to characterize adverse events that cannot be described by discharge fields on the hospital chargemaster; limiting adverse events by pharmacologic reversal agents and mortality certainly underestimates to total number of adverse events but appropriately characterizes those serious adverse events that may have longstanding patient or hospital implications. However, for questions such as these, the ability to conduct a well-controlled, randomized study of such a size to provide statistically relevant results is limited by ethical considerations and standard of care guidelines that now recommend the use of capnography in these types of procedures. The cost of performing such a study is also likely to be prohibitive. Despite the inherent limitations of a database analysis, we believe that our results are strengthened by the large sample size and the use of propensity score matching to generate well-matched patient populations.

Another concern with a retrospective database analysis lies in the use CPT/ICD-9 codes to accurately identify patients of interest and to effectively capture the use of specific patient monitoring equipment. It is important to note that our analysis only reports events observed in the two patient populations (capnography $\pm \mathrm{SpO}_{2}$ vs. $\mathrm{SpO}_{2}$ only) for whom patient monitoring was reported and that we did not infer any conclusions from the patient population for which no monitoring was reported. We recognize that the relatively large group of patients for whom neither $\mathrm{SpO}_{2}$ nor capnography monitoring were reported may be improbable, but given our reliance on billing codes to identify the monitoring equipment utilized, cases involving reusable sensors that were not included in the billing record would have been omitted from the analysis. Given the availability of reusable $\mathrm{SpO}_{2}$ sensors, it is likely that our analysis underestimates the use of $\mathrm{SpO}_{2}$ monitoring. It is in part due to this limitation that we grouped capnography only patients with capnography plus $\mathrm{SpO}_{2}$ patients in our final analysis, in that we find it unlikely that a patient would receive capnography monitoring without $\mathrm{SpO}_{2}$ monitoring. While there are known methods of $\mathrm{CO}_{2}$ sampling that do not require a specific sensor, the use of these technologies was more common prior to the availability of capnography-specific patient interface sensors (which do not utilize reusable sensors), thus errors of omission with respect to capnography monitoring are less likely.

Despite these limitations regarding sensor use, we believe that the combination of procedural and diagnostic codes employed was optimized for the current analysis. Also, due to the discharge-level nature of the data contained in the Premier database, we cannot directly comment on the specific timing of patient monitoring and/ or medication administration relative to the GEP of interest. An additional limitation of the Premier database is that certain variables, including patient BMI and history of comorbidities such as sleep apnea, are not captured in the database and thus are not available for inclusion in the propensity score calculations, which might potentially impact the estimation of the capnography effect.

\section{Implications of findings}

The addition of capnography to procedural monitoring without understanding how to optimize the information it provides is unlikely to be very useful. The Centers for Medicare and Medicaid Services (CMS) Conditions of Participation requires that all anesthesia services (whether administered by an anesthesia-trained provider or not) in a hospital facility be organized under the directorship of an anesthesiologist. This study may help provide the necessary information to encourage appropriate monitoring standards in gastrointestinal (GI) procedure suites and to encourage and assist in providing the necessary education to utilize data from the patient monitoring devices to more effectively manage patients receiving procedural sedation. In our era of increased public awareness of patient safety events, we must guard against a normalization of deviance when dealing with relatively rare but clinically significant events. To provide safe care and minimize the potential for preventable harm, we must continue to learn from the mistakes of others to help ensure the best reasonable outcome from these extremely common procedures.

The addition of electronic monitoring or physician work to any procedure may be perceived as costadditive. One recent study reported that the addition of capnography to an endoscopy procedural sedation monitoring protocol resulted in a $27.2 \%$ and $18.0 \%$ reduction in the proportion of patients experiencing an adverse event during deep and moderate procedural sedation/analgesia, respectively [29]. For this analysis, the authors reported that the median number needed to treat to avoid any adverse event was 8 patients for deep sedation and 6 patients for moderate sedation, and estimated that the addition of capnography reduced the cost per procedure by $\$ 85$ (during deep sedation) and $\$ 35$ (during moderate sedation). The authors concluded that capnography is estimated to be cost-effective if not cost-saving during procedural sedation for gastrointestinal endoscopy and suggested that the addition of capnography monitoring to the standard of care during procedural sedation for endoscopy should be considered.

\section{Conclusions}

The results of our analysis indicate that the use of capnography during GEP with procedural sedation is 
associated with significant reductions in the risk of pharmacological rescue events in outpatients and death in inpatients. Despite the limitations of this retrospective data-based study, we believe the use of capnography during GEP performed with sedation should be recommended.

\section{Additional files}

Additional file 1: Table S1. Current Procedural Terminology Codes for Esophagoscopy, Small Bowel Endoscopy, Colonoscopy, Sigmoidoscopy and Anoscopy Procedures. (DOCX $188 \mathrm{~kb}$ )

Additional file 2: Table S2. Capnography Sensor Codes. (DOCX 178 kb) Additional file 3: Table S3. Variables Included in the Multivariable Logistic Regression. (DOCX 67 kb)

Additional file 4: Table S4. Propensity Score Matching - Inpatient Population. (DOCX $170 \mathrm{~kb}$ )

Additional file 5: Table S5. Propensity Score Matching - Outpatient Population. (DOCX $155 \mathrm{~kb}$ )

Additional file 6: Table S6. Patient Outcomes Before and After Propensity Score Matching - Inpatient Population. (DOCX 63 kb)

Additional file 7: Table S7. Patient Outcomes Before and After Propensity Score Matching - Outpatient Population. (DOCX 63 kb)

\section{Abbreviations}

ASA: American Society of Anesthesiologists; CCl: Charlson Comorbidity Index CFR: Code of Federal Regulations; Cl: Confidence interval; CMS: Centers for Medicare and Medicaid Senvices; COPD: Chronic obstructive pulmonary disease; CPT: Current Procedural Terminology; EGD: Esophagogastroduodenoscopy; ERCP: Endoscopic retrograde cholangiopancreatography; $\mathrm{ETCO}_{2}$ : End-tidal carbon dioxide; GEP: Gastrointestinal endoscopic procedures; Gl: Gastrointestinal; HIPAA: Health Insurance Portability and Accountability Act; ICD-9CM: International Classification of Diseases 9th revision Clinical Modification; IRB: Institutional review board; OR: Odds ratio; PS: Propensity Score; $\mathrm{SaO}_{2}$ : Arterial oxygen saturation; SD: Standard deviation; $\mathrm{SpO}_{2}$ : Pulse oximetry

\section{Acknowledgements}

The authors acknowledge Brooke Harrison, PhD for technical assistance during the preparation of this manuscript.

\section{Funding}

This analysis was supported by Medtronic. Medtronic had no role in the design of the study and collection, analysis, and interpretation of data and in writing the manuscript.

\section{Availability of data and materials}

The data that support the findings of this study are available from Premier Inc. but restrictions apply to the availability of these data, which were used under license for the current study, and so are not publicly available. Data are however available from the authors upon reasonable request and with permission of Premier Inc.

\section{Authors' contributions}

$J \mathrm{Q}$ analyzed the data; MWJ and JQ designed the study, interpreted the data, and wrote the manuscript. Both authors read and approved the final manuscript.

\section{Ethics approval and consent to participate}

Per United States Title 45 CFR (Code of Federal Regulations) Part 46.101, institutional review board (IRB) approval for this study was not required under the exemption that this research involved the study of existing data and that the information was recorded in such a manner that the subjects could not be identified, directly or through identifiers linked to the subjects. Additional detail regarding the data provided by Premier Inc. is provided below (see Availability of data and material).
Consent for publication

Not applicable.

\section{Competing interests}

MWJ has received grant support and honoraria from Covidien/Medtronic; JQ is an employee of Medtronic.

\section{Publisher's Note}

Springer Nature remains neutral with regard to jurisdictional claims in published maps and institutional affiliations.

\section{Author details}

${ }^{1}$ NorthStar Anesthesia, Springfield Regional Medical Center, Springfield, $\mathrm{OH}_{4}$ USA. ${ }^{2}$ Health Economics and Outcomes Research, Medtronic, Mansfield, MA, USA.

Received: 8 May 2017 Accepted: 21 November 2017

Published online: 28 November 2017

\section{References}

1. Beitz A, Riphaus A, Meining A, Kronshage T, Geist C, Wagenpfeil S, et al. Capnographic monitoring reduces the incidence of arterial oxygen desaturation and hypoxemia during propofol sedation for colonoscopy: a randomized, controlled study (colocap study). Am J Gastroenterol. 2012;107: 1205-12.

2. Ramsay MA, Newman KB, Jacobson RM, Richardson $C T$, Rogers L, Brown BJ, et al. Sedation levels during propofol administration for outpatient colonoscopies. Proc (Bayl Univ Med Cent). 2014:27:12-5.

3. Hart R, Classen M. Complications of diagnostic gastrointestinal endoscopy Endoscopy. 1990;22:229-33.

4. Arrowsmith JB, Gerstman BB, Fleischer DE, Benjamin SB. Results from the American Society for Gastrointestinal Endoscopy/U.S. Food and Drug Administration collaborative study on complication rates and drug use during gastrointestinal endoscopy. Gastrointest Endosc. 1991:37:421-7.

5. Daneshmend TK, Bell GD, Logan RF. Sedation for upper gastrointestinal endoscopy: results of a nationwide survey. Gut. 1991:32:12-5.

6. Sharma VK, Nguyen CC, Crowell MD, Lieberman DA, de Garmo P, Fleischer DE. A national study of cardiopulmonary unplanned events after $\mathrm{Gl}$ endoscopy. Gastrointest Endosc. 2007;66:27-34.

7. Dumonceau JM, Riphaus A, Aparicio JR, Beilenhoff U, Knape JT, Ortmann M, et al. European Society of Gastrointestinal Endoscopy, European Society of Gastroenterology and Endoscopy Nurses and associates, and the European Society of Anaesthesiology Guideline: non-anaesthesiologist administration of propofol for gi endoscopy. Eur J Anaesthesiol. 2010;27:1016-30.

8. American Society of Anesthesiologists Task Force on, Sedation Analgesia by, Non-Anesthesiologists. Practice guidelines for sedation and analgesia by non-anesthesiologists. Anesthesiology. 2002;96:1004-17.

9. Vargo JJ, Zuccaro G Jr, Dumot JA, Conwell DL, Morrow JB, Shay SS. Automated graphic assessment of respiratory activity is superior to pulse oximetry and visual assessment for the detection of early respiratory depression during therapeutic upper endoscopy. Gastrointest Endosc. 2002; 55:826-31.

10. Burton JH, Harrah JD, Germann CA, Dillon DC. Does end-tidal carbon dioxide monitoring detect respiratory events prior to current sedation monitoring practices? Acad Emerg Med. 2006;13:500-4.

11. Metzner J, Posner KL, Domino KB. The risk and safety of anesthesia at remote locations: the US closed claims analysis. Curr Opin Anaesthesiol. 2009:22:502-8.

12. Qadeer MA, Vargo JJ, Dumot JA, Lopez R, Trolli PA, Stevens T, et al. Capnographic monitoring of respiratory activity improves safety of sedation for endoscopic cholangiopancreatography and ultrasonography. Gastroenterology. 2009;136:1568-76.

13. Lightdale JR, Goldmann DA, Feldman HA, Newburg AR, DiNardo JA, Fox VL. Microstream capnography improves patient monitoring during moderate sedation: a randomized, controlled trial. Pediatrics. 2006;117:e1170-8.

14. Friedrich-Rust M, Welte M, Welte C, Albert J, Meckbach Y, Herrmann E, et al. Capnographic monitoring of propofol-based sedation during colonoscopy. Endoscopy. 2014:46:236-44.

15. Casati A, Gallioli G, Scandroglio M, Passaretta R, Borghi B, Torri G. Accuracy of end-tidal carbon dioxide monitoring using the NBP-75 microstream 
capnometer. A study in intubated ventilated and spontaneously breathing nonintubated patients. Eur J Anaesthesiol. 2000;17:622-6.

16. Kasuya Y, Akca O, Sessler DI, Ozaki M, Komatsu R. Accuracy of postoperative end-tidal $\mathrm{pCO}_{2}$ measurements with mainstream and sidestream capnography in non-obese patients and in obese patients with and without obstructive sleep apnea. Anesthesiology. 2009;111:609-15.

17. Krauss B, Hess DR. Capnography for procedural sedation and analgesia in the emergency department. Ann Emerg Med. 2007;50:172-81.

18. Stokes ME, Ye X, Shah M, Mercaldi K, Reynolds MW, Rupnow MF, et al. Impact of bleeding-related complications and/or blood product transfusions on hospital costs in inpatient surgical patients. BMC Health Serv Res. 2011;11:135.

19. Parsons LS. Reducing bias in a propensity score matched-pair sample using greedy matching techniques. SUGI 26 Proceedings 2001

20. Austin PC. Balance diagnostics for comparing the distribution of baseline covariates between treatment groups in propensity-score matched samples. Stat Med. 2009;28:3083-107.

21. Tobias JD, Leder M. Procedural sedation: a review of sedative agents, monitoring, and management of complications. Saudi J Anaesth. 2011;5:395-410.

22. Pino RM. The nature of anesthesia and procedural sedation outside of the operating room. Curr Opin Anaesthesiol. 2007;20:347-51.

23. Arakawa H, Kaise M, Sumiyama K, Saito S, Suzuki T, Tajiri H. Does pulse oximetry accurately monitor a patient's ventilation during sedated endoscopy under oxygen supplementation? Singap Med J. 2013;54:212-5.

24. Bhananker SM, Posner KL, Cheney FW, Caplan RA, Lee LA, Domino KB. Injury and liability associated with monitored anesthesia care: a closed claims analysis. Anesthesiology. 2006;104:228-34.

25. Anderson JL, Junkins E, Pribble C, Guenther E. Capnography and depth of sedation during propofol sedation in children. Ann Emerg Med. 2007:49:9-13.

26. Deitch K, Miner J, Chudnofsky CR, Dominici P, Latta D. Does end tidal $\mathrm{CO}_{2}$ monitoring during emergency department procedural sedation and analgesia with propofol decrease the incidence of hypoxic events? A randomized, controlled trial. Ann Emerg Med. 2010;55:258-64.

27. Deitch K, Chudnofsky CR, Dominici P. The utility of supplemental oxygen during emergency department procedural sedation with propofol: a randomized, controlled trial. Ann Emerg Med. 2008:52:1-8.

28. ASA Standards for Basic Anesthetic Monitoring, Committee of Origin: Standards and Practice Parameters. http://www.asahq.org/ /media/sites/ asahq/files/public/resources/standards-guidelines/standards-for-basicanesthetic-monitoring.pdf. Accessed Mar 2017.

29. Saunders R, Erslon M, Vargo J. Modeling the costs and benefits of capnography monitoring during procedural sedation for gastrointestinal endoscopy. Endosc Int Open. 2016;4:E340-51.

30. Jopling M, Trost D, Kofol T, Warner E. Capnography monitoring in procedural sedation: a hospital-wide cost-avoidance model. Presented at 24th Society for Technology in Anesthesia (STA) Annual Meeting. January 7-10, 2015, Phoenix, Arizona. https://www.stahq.org/files/2214/4535/8382/ 19_Abstract_Jopling.pdf. Accessed Nov 2017.

\section{Submit your next manuscript to BioMed Central and we will help you at every step:}

- We accept pre-submission inquiries

- Our selector tool helps you to find the most relevant journal

- We provide round the clock customer support

- Convenient online submission

- Thorough peer review

- Inclusion in PubMed and all major indexing services

- Maximum visibility for your research

Submit your manuscript at www.biomedcentral.com/submit

Biomed Central 